\title{
Severe plastic deformation by incremental angular splitting
}

\author{
Andrzej Rosochowski • Malgorzata Rosochowska • \\ Lech Olejnik
}

Received: 11 November 2012/ Accepted: 21 December 2012/Published online: 5 January 2013

(C) The Author(s) 2013. This article is published with open access at Springerlink.com

\begin{abstract}
A new way of generating large plastic strain in a billet by splitting it plastically by a reciprocating punch is investigated. The concept of incremental angular splitting (I-AS) is explained by referring to the severe plastic deformation process of incremental equal channel angular pressing (I-ECAP). Results of laboratory trials of I-ECAP and I-AS, using a purposely designed AA1070 billet, are presented and both processes are compared. Further comparison is based on Vickers hardness distribution in the billet subjected to I-ECAP and I-AS. FE simulation results give an indication of strain distribution in both processes. The main advantages of I-AS appear to be more flexibility in the billet choice and more uniform strain distribution in the first pass of the process. A possibility of creating a gradient material by I-AS with a flat punch is considered.
\end{abstract}

\section{Introduction}

Bulk metals with ultrafine grained (UFG) structure, characterized by the average grain size $<1 \mu \mathrm{m}$, draw substantial

\footnotetext{
A. Rosochowski $(\bowtie)$

Design, Manufacture and Engineering Management, University of Strathclyde, James Weir Building, 75 Montrose Street, Glasgow G1 1XJ, UK

e-mail: a.rosochowski@strath.ac.uk

M. Rosochowska

Advanced Forming Research Centre, University of Strathclyde, 85 Inchinnan Drive, Renfrew PA4 9LJ, UK

e-mail: m.rosochowska@strath.ac.uk

L. Olejnik

Institute of Manufacturing Processes, Warsaw University of Technology, ul. Narbutta 85, 02-524 Warsaw, Poland e-mail: 1.olejnik@wip.pw.edu.pl
}

attention due to their unique mechanical and physical properties. The preferable method of producing bulk UFG metals is severe plastic deformation (SPD) [1]. In this method, a very large plastic deformation (true strain 3-10 depending on the metal) "subdivides" coarse metal grains into sub-micrometer size grains. The nature of grain refinement and its consequences for material properties have been very popular research areas especially for the last 10 years. Less explored seem to be SPD processes, which are necessary to produce the UFG structure. There are many SPD processes used on the laboratory scale, but only few have a chance to become industrially viable. The most popular batch SPD processes are equal channel angular pressing (ECAP) of short bars [2] and high pressure torsion of thin discs [3]. From the manufacturing point of view, more promising are SPD processes dealing with long bars, plates and sheets. For example, ECAP-Conform can be used for long bars [4] while accumulated roll bonding for sheets [5]. Incremental ECAP (I-ECAP), which was introduced by the authors [6], is suitable for all three billet forms [7]. However, a great variety of material shapes makes it sometimes difficult to follow the exact definition of SPD as a method, which does not change the billet shape and dimensions. For example, this is the case with long tubes processed by I-ECAP, where the wall thickness of the tube remains unchanged but its diameter does change [8]. On the other hand, there are manufacturing processes whose purpose is to change the billet shape but the way it is achieved has a lot in common with ECAP. One of those processes is machining by cutting. It has been long recognized that chip creation during cutting is due to simple shear like in ECAP. Figure 1, which has been obtained by finite element (FE) simulation of orthogonal cutting using Deform software, illustrates this point. It can be seen that strain distribution is not uniform; 


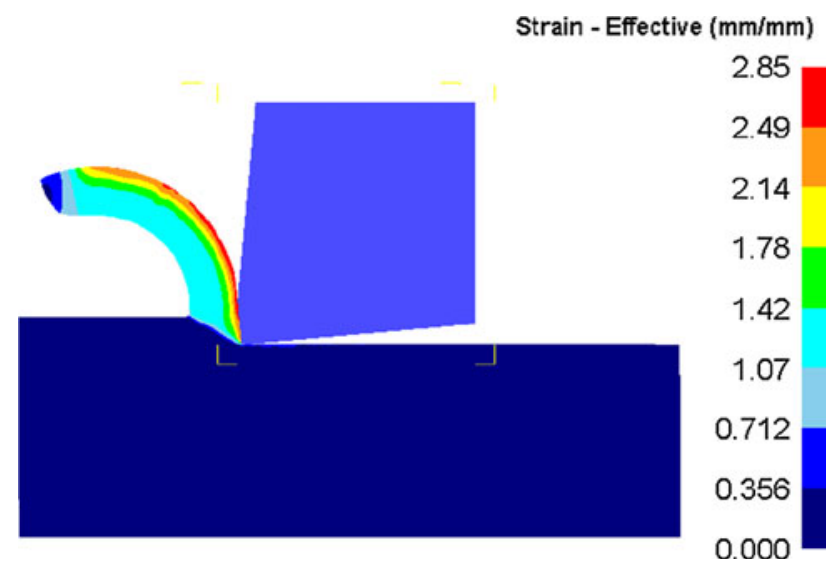

Fig. 1 Strain distribution in orthogonal cutting established by FE simulation using Deform FE software

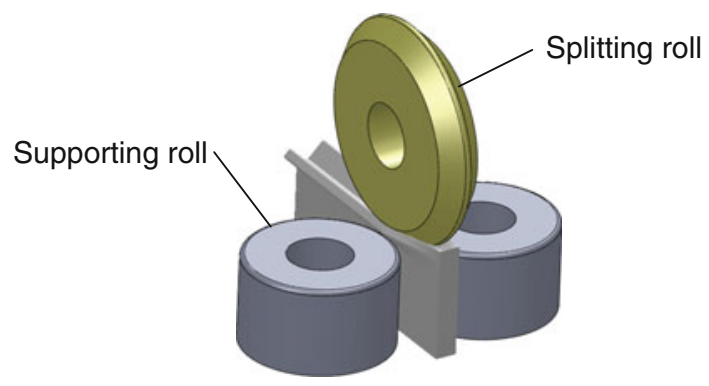

Fig. 2 Configuration of tools in linear flow splitting of plate

the largest strain is on the tool side of the chip. Similar results have been obtained by FE simulation of cutting using Abaqus software [9]; Ref. [9] is one of a number of publications exploring a possibility of creating UFG structure by cutting.

Another example of a shaping process related to ECAP is linear flow splitting [10]. It was invented to create long structural $\mathrm{Y}$ cross section profiles from straight plates (Fig. 2). To initiate this process, a plate is put between two cylindrical rolls, with one end of the plate sticking slightly out. A V-shaped roll presses the edge of the plate outside the cylindrical rolls, which causes the plate to split plastically. The geometry of the rolls depends on the required profile of the Y split. An additional effect of this process is grain refinement resulting from strain concentration on the surface of Y profile, which is in contact with a splitting roll.

The above examples suggest that there still might be "undiscovered" SPD and other plastic deformation processes potentially useful for refining grain structure using a variety of process configurations and billet shapes. The inspiration for this paper was a process of double billet I-ECAP [11], in which two billets would be replaced with a single billet split plastically in half, with a view to refine its grain structure. The new process, called incremental

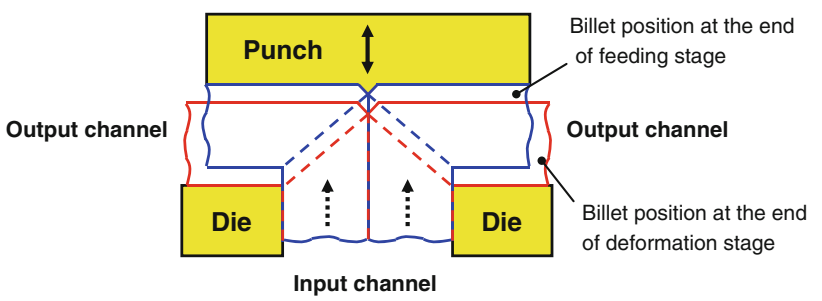

Fig. 3 Incremental ECAP with two billets fed incrementally through the input channel and deformed plastically by a reciprocating punch

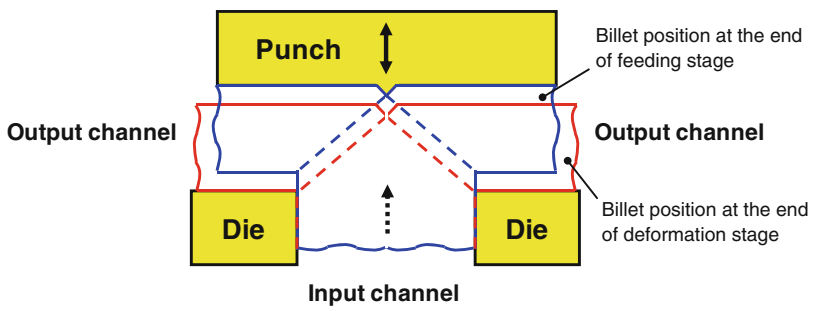

Fig. 4 Incremental AS with one billets fed incrementally through the input channel and split plastically by a reciprocating punch

angular splitting (I-AS), was proposed in [12] but since the experimental results raised some doubts, it was necessary to carry out a more robust experiment. This paper presents results of such an experiment and the analysis of the material flow during I-AS carried out by FE simulation.

\section{Concept of I-AS}

The concept of I-AS originated from a version of I-ECAP, which processes two square cross-section parallel billets put into a common channel [11]. The billets are fed incrementally to present the material to a reciprocating punch, which causes plastic deformation. Figure 3 shows the positions of billets at the beginning and at the end of each deformation cycle, which was proved in [6] to result in simple shear of a dashed region as observed in classical ECAP; the punch is shown in the upper position. Since feeding and deformation occur at different times, the feeding force can be substantially reduced compared to the situation when feeding causes plastic deformation like in classical ECAP. The punch is equipped with a central spike, which facilitates material flow into the output channel.

The only change required for the above process to become I-AS [12] is the replacement of two parallel billets in the input channel with only one billet having a rectangular cross section equal to the sum of cross sections of the output channels (Fig. 4). The punch face plays the role of a splitting tool, which divides the billet into two equal billets and forces them into the output channels. It has to be emphasized that the splitting process is plastic in nature 
(a)

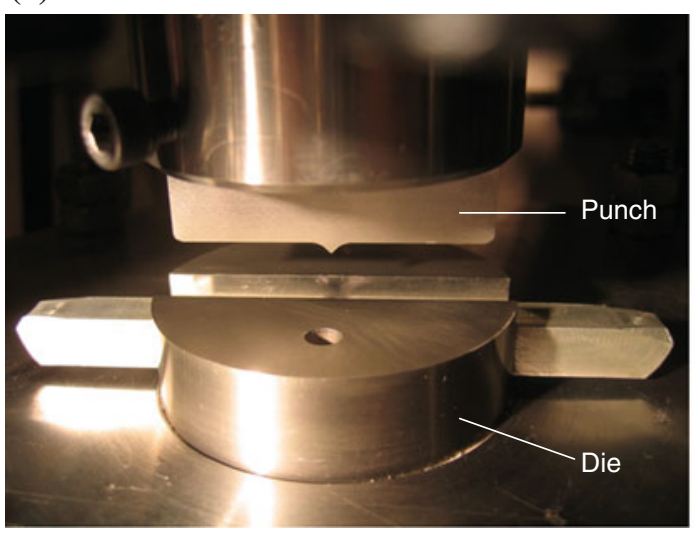

(b)

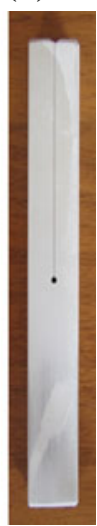

Fig. 5 I-ECAP device used for I-AS (a) and AA1070 aluminum billet precut approximately half its length (b)

(no fracture) and therefore similar to plastic cutting. One can say that it creates a bridge between cutting and ECAP. However, in order to be able to process long billets, an incremental version of ECAP is considered.

\section{Experiment}

Because of its origin, I-AS could be easily performed using the tools and machine used earlier for I-ECAP (Fig. 5a). The process was carried out at room temperature; the punch sine wave frequency was $1 \mathrm{~Hz}$, the peak-to-peak amplitude was $1.6 \mathrm{~mm}$, and the feeding stroke was $0.2 \mathrm{~mm}$. To avoid any doubts regarding the process conditions and the source of material when comparing both processes, it was decided to combine I-ECAP and I-AS in one experiment. This was achieved by designing an AA1070 aluminum billet, which had a rectangular cross section $10 \mathrm{~mm} \times 20 \mathrm{~mm}$ and was precut along approximately half its length by wire EDM (Fig. 5b). The billet was lubricated with $\mathrm{MoS}_{2}$ applied to a conversion coat of calcium aluminate created earlier.

Since the billet was orientated in the input channel in the way shown in Fig. 5b, the first process to occur was I-ECAP. After reaching the precut end hole, the punch started splitting the material plastically; I-AS continued until the last portion of billet was fed. Figure 6 shows the result of this process. The transition from I-ECAP to I-AS is clearly visible as the precut end hole left marks. The quality of top surface produced by I-ECAP was better than that produced by I-AS despite the lack of lubrication in both cases; the I-ECAPed part was not lubricated because precutting took place after conversion coating and, in addition, the precut gap was not wide enough to apply the lubricant. The flash caused by a clearance between the

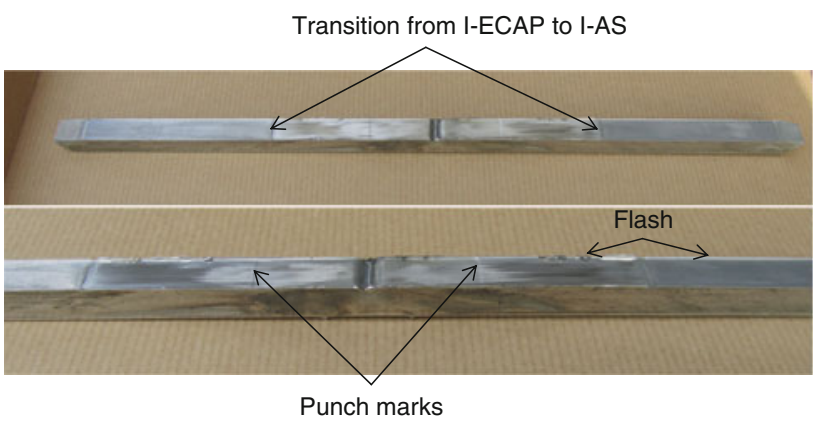

Fig. 6 Split billet after I-ECAP followed by I-AS (top) and its enlarged view (bottom)

punch and the die was more pronounced in the case of I-AS. The punch marks could have been avoided if better control was exercised over the feeding force causing the process to stop (an automatic stop has been used).

The punch force and the feeder force were recorded during the whole process (Fig. 7). The punch force varied between its maximum value and zero in each cycle. The feeder force varied between a top envelope, when the feeder was stationary and supporting the billet subjected to plastic deformation and a bottom envelope, when the feeder was feeding the billet as a rigid body. A gap in the recorded punch force indicates those few cycles, during which the punch was going through the precut end hole. The analysis of the recorded forces does not reveal any substantial difference in their nature or value resulting from the process change from I-ECAP to I-AS. The visible graduate changes are similar to those recorded for I-ECAP only and result from a decreasing friction force in the input channel as the billet length in this channel decreases.

To characterize material changes resulting from one pass of I-ECAP/I-AS, Vickers hardness HV0.1 was measured along one of the billets/split billets as well as at five locations in the transverse direction. The results of these measurements are shown in Fig. 8. The longitudinal distribution of hardness is non-uniform, with the lowest value approaching the original hardness of HV32 at both ends, which results from the lack of plastic deformation there. The maximum hardness achieved is approximately HV55 for the I-ECAP side and HV50 for the I-AS side; in the middle part of the billet, the hardness goes down to about HV40. There is no sudden change of this hardness at the point of transition from I-ECAP to I-AS. However, the transverse distribution of hardness is different for the three locations on the I-ECAP side and the two locations on the I-AS side. While the I-ECAPed billet shows a lower value of hardness on the punch side $(<\mathrm{HV} 40$ at the transverse coordinate 0 ), the I-ASed billet displays more uniform distribution in the transverse direction. 
Fig. 7 Forces on the punch and the feeder during I-ECAP followed by I-AS (time axis has been reversed)

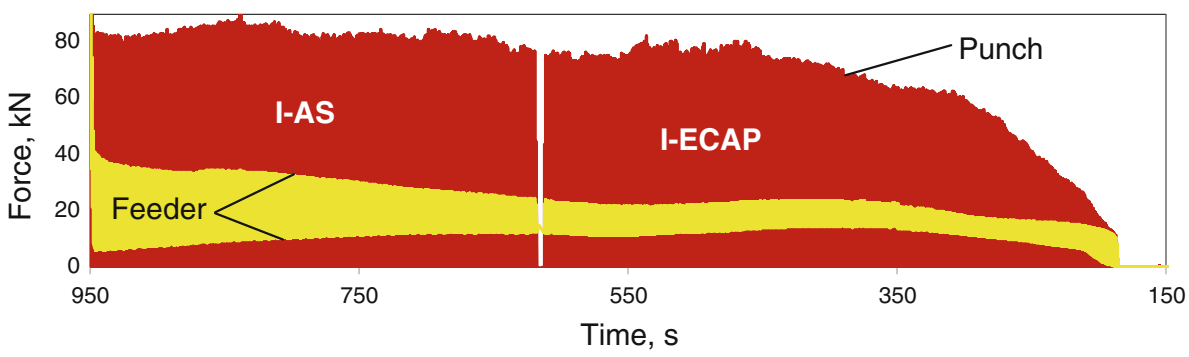

Fig. 8 Vickers hardness HV0.1 measured along billet subjected to I-ECAP and I-AS and at five locations in the transverse direction

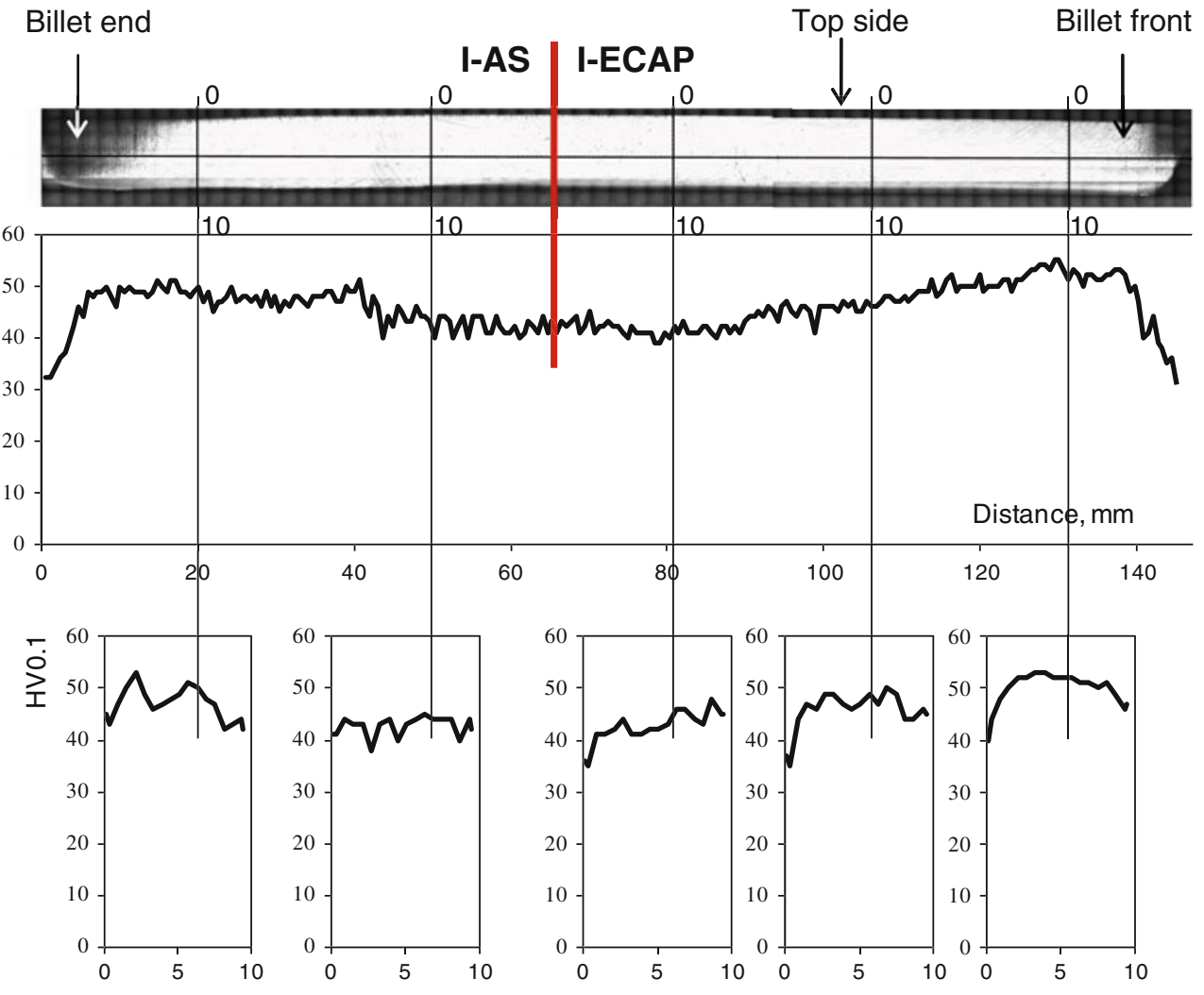

\section{FE simulation}

FE simulation of cutting has been reported in numerous publications. However, it is not trivial because of large distortion of finite elements caused by severe deformation generated in the cut chip. The only way to avoid numerical problems is to use remeshing. A popular FE program, Abaqus, has adaptive meshing, which, however difficult to use, can lead to reasonable results [9]. An easier approach is to rely on the automatic remeshing capability offered by Deform, which was used in this research.

The material model used in the analysis was a rigid plastic, von Mises material with strain hardening $(\sigma=159$ $\left.(0.02+\varepsilon)^{0.27}\right)$ established by compression testing of a standard, coarse grained AA1070. The Coulomb's friction coefficient was assumed to be 0.1 . The model geometry as well as most process parameters were the same as in the experiment; only the punch amplitude was reduced compared to the experiment to shorten computational time. The mesh of free form quadrilateral elements was automatically generated, but was refined in a predefined area below the punch. The same area was used to undertake remeshing during simulation. Since the process analyzed was plain strain, Deform 2D was used for the simulation.

The first simulation was for I-ECAP to compare the results obtained by Deform to those obtained earlier by Abaqus [7]. Figure 9a displays Deform results in terms of equivalent/effective strain. The distribution of strain obtained by two programs appeared to be similar. The second simulation was for I-AS (Fig. 9b) and showed a different strain distribution, with substantially larger strain on the punch side of the billet. The third simulation was for I-ECAP followed by I-AS, as in the experiment (Fig. 9c). Strain distribution in this case follows approximately the 
Fig. 9 Equivalent strain distribution for I-ECAP (a), I-AS (b) and I-ECAP followed by I-AS (c)

(a)

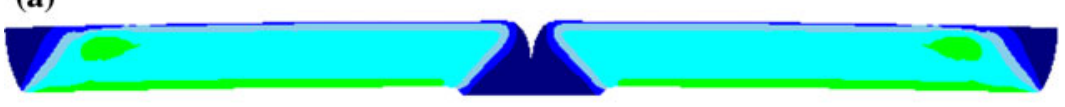

(b)

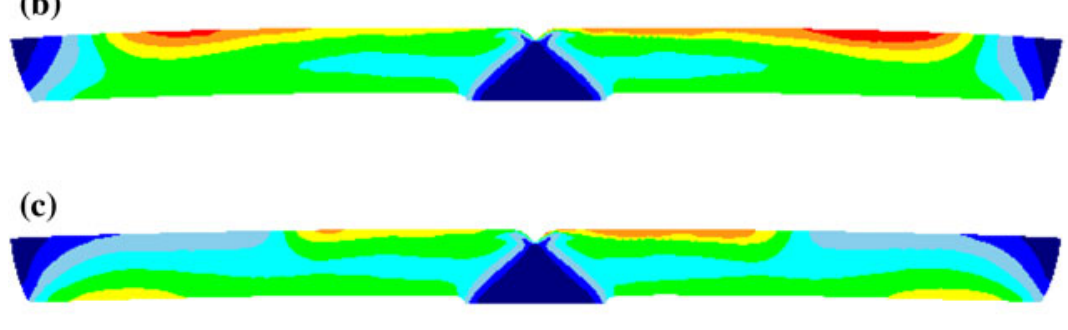

Fig. 10 Equivalent strain distribution for I-AS with a flat punch

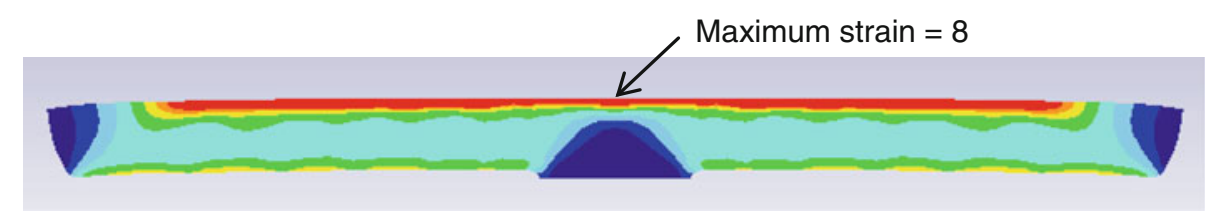

distributions identified for I-ECAP (any difference here may be due to the thickness of the precut gap) and I-AS in the respective parts of the billet.

Strain distribution in the I-ASed billet depends on the punch geometry. The geometry alteration tested here was a flat punch without a spike. Figure 10 displays strain distribution for this case. The strain scale is the same as in Fig. 9, which means that the highest equivalent strain of approximately 8 on the billet surface facing the punch cannot be distinguished from the maximum strain of 2.3. This has been done deliberately to show the areas with lower strain.

\section{Discussion}

Since I-AS is a new process, for which there is no previous data, the reliability of results presented should be discussed. The experiment has been made insensitive to a possible material and process parameter inconsistence because the same billet was subjected to both I-ECAP and I-AS in one process. The punch force and the feeder force for I-ECAP and I-AS were also measured in one process. It was a bit surprising that these forces did not show any abrupt change when I-ECAP became I-AS, but this may only mean that any difference between the two processes is very local while forces tend to describe a global behavior in terms of material flow and tool friction.

Vickers hardness distribution results have shed some light on those differences indicating increased hardness on the punch side of the billet for the material processed by I-AS. A puzzling result, but independent on the type of process, was lower hardness along the billet in its middle part. The question remains whether this lower hardness is an anomaly or perhaps the higher hardness observed at both ends should be treated as an anomaly. Using longer billets should give an answer to this. Generally, a more robust Vickers hardness measuring technique should be used to reveal details of hardness concentration close to the billet surface. This could involve embedding or fixing billet in a supporting block, better quality surface polishing and reducing the indentation force to decrease the size of indents.

FE simulation is a useful tool for revealing strain distribution, but it can still be problematic in terms of the quality of input data and numerical procedures used by the FE programs. Strain hardening data used in Deform were established in a compression test carried out till the strain of 1.5. This means that for higher strains, the yield stress was extrapolated. It was probably alright for processes displayed in Fig. 9, for which the maximum strain did not exceed 2.3 but it was rather questionable in the case of flat punch (Fig. 10), for which the strain of 8 was reached. It is also uncertain what value of friction coefficient should be used, especially at the punch billet interface in I-AS. The quality of remeshing is also a critical factor, which has to be evaluated. One indication of the possible problems is the lack of symmetry of strain distribution in Fig. $9 \mathrm{~b}$ and $\mathrm{c}$ resulting from a non-symmetric character of remeshing. It is recommended that different $\mathrm{FE}$ programs are used to compare the remeshing results.

I-AS appears to be similar to I-ECAP and, therefore, to classical ECAP in terms of material flow and the resulting hardness in the bulk of the billet, except the top (punch) side of the billet where strain and hardness are more uniform. This convinced the authors that I-AS should lead to a 
similar grain refinement as in ECAP and I-ECAP. Consequently, it has been decided not to check the microstructure of AA1070 after I-AS because the same material was investigated in many earlier publications (interesting TEM images has been published recently for AA1070 subjected to one pass of ECAP with converging billets [13]).

Looking at the benefits of I-AS, it increases the choice of billet shapes by giving an option to plastically split a billet into two billets. I-AS generates a higher strain in the vicinity of the split surface, which leads to more uniform hardness distribution in the transverse direction compared to I-ECAP and ECAP. In the special case of I-AS with a flat punch, a very high strain and possibly high hardness could be achieved in this region. In most cases, when uniform billets are desirable, this will be inappropriate. However, for gradient materials, surface strengthening resulting from I-AS with a flat punch may open up new possibilities. This option will be further investigated.

\section{Conclusions}

- A new process called I-AS has been proposed to explore the possibility of generating SPD using plastic separation of the material as it is known for cutting. Thus, I-AS is a link between cutting and ECAP (I-ECAP), which have traditionally been approached separately.

- I-AS was realized using a laboratory rig used earlier for I-ECAP of two billets. This means that no investment for new equipment was necessary.

- The only change required was a different billet; a special billet was designed for the experiment to enable I-AS and I-ECAP to be performed on one billet and using the same process parameters. This made the comparison of both processes more reliable.

- The billets produced by I-AS were geometrically similar to those obtained by I-ECAP.

- The punch force and the feeder force in I-AS were similar to those observed in I-ECAP.

- Vickers hardness distribution in I-AS was similar to that in I-ECAP except a locally increased hardness beneath the split surface. This makes hardness distribution more uniform across the billet compared to I-ECAP, which suffers form much lower strain in this area.

- The new process can be used to reduce the crosssection area of bulk billets while refining their grain structure (UFG structure assumption based on strain and hardness distribution).

- I-AS with a flat punch should be able to produce a thin layer of highly strained and strengthened material on the top (punch) side of the billet, which could be useful for creating gradient materials. This possibility will be the subject of further investigation.

Acknowledgements Part of this research was supported by the Engineering and Physical Sciences Research Council [grant number EP/G03477X/1].

Open Access This article is distributed under the terms of the Creative Commons Attribution License which permits any use, distribution, and reproduction in any medium, provided the original author(s) and the source are credited.

\section{References}

1. Azushima A, Kopp R, Korhonen A, Yang DY, Micari F, Lahoti GD, Groche P, Yanagimoto J, Tsuji N, Rosochowski A, Yanagida A (2008) CIRP Ann Manuf Technol 57:716

2. Segal VM, Reznikov VA, Drobyshevskiy AE, Kopylov VI (1981) Russ Metall 1:99

3. Valiev RZ, Krasilnikov NA, Tsenev NK (1991) Mater Sci Eng A 137:35

4. Raab GJ, Valiev RZ, Lowe TC, Zhu YT (2004) Mater Sci Eng A 382:30

5. Saito Y, Tsuji N, Utsunomiya H, Sakai T, Hong RG (1998) Scripta Mater 39:1221

6. Rosochowski A, Olejnik L (2007) AIP Conf Proc 907:653

7. Rosochowski A, Olejnik L (2010) Mater Sci Forum 674:19

8. Rosochowski A, Olejnik L (2011) AIP Conf Proc 1353:517

9. Sevier M, Lee S, Shankar MR, Yang HTY, Chandrasekar S, Compton WD (2006) Mater Sci Forum 503-504:3798

10. Groche P, Vucic D, Jockel M (2007) J Mater Process Technol 183:249

11. Rosochowski A, Olejnik L, Richert M (2008) Mater Sci Forum 584-586:139

12. Rosochowski A, Olejnik L (2012) Key Eng Mater 504-506:569

13. Rosochowski A, Olejnik L, Richert J, Rosochowska M, Richert M (2013) Mater Sci Eng A 560:358 